\title{
Blunt Injury to the Aorta, Esophagus, and Diaphragm
}

\author{
Daniel Lollar ${ }^{1}$ - Gregory J. Jurkovich ${ }^{1}$
}

Published online: 16 October 2015

(C) Springer International Publishing AG 2015

\begin{abstract}
Blunt injury to the aorta, diaphragm, and esophagus are unusual and are typically the result of a significant transfer of energy and hence are typically accompanied by other injuries. The associated injury can detract attention, leading to lethal delays in the recognition of blunt injury to the aorta, esophagus, and diaphragm. Improved cross-sectional imaging techniques (CT scanning) and a better understanding of the subtle signs of blunt injury to the aorta, diaphragm, and even esophagus has lessened, but not eliminated, the delay in diagnosis. Changes in management of aortic injuries continue to involve, largely stimulated by the more widespread application of endovascular techniques, and a better understanding of the natural history of less severe grades of injury. Early and primary repair of an acute blunt diaphragm injury remains the standard. Blunt esophageal injury, perhaps the rarest in this category, can largely be excluded on the basis of a detailed thoracic CT scan, although the role of esophageal endoscopy or esophagography remains essential diagnostic tools. The management of these rare injuries is often controversial. This review will examine the recent literature regarding these uncommon injuries, emphasizing recent clinical trials and guidelines.
\end{abstract}

Keywords Incidence $\cdot$ Diagnosis $\cdot$ Blunt trauma $\cdot$ Computed tomography of chest - Aortography · Aortic injury .

Endovascular procedures · Cardiovascular surgery .

This article is part of the Topical Collection on Blunt Trauma to the Chest

Daniel Lollar

daniel.lollar@ucdenver.edu

Gregory J. Jurkovich

jerryj@dhha.org

1 Denver Health Medical Center, 777 Bannock St. MC0206, Denver, CO 80204, USA
Diaphragm injury $\cdot$ Laparoscopic surgery $\cdot$ Esophageal perforation $\cdot$ Open operations

\section{Introduction}

\section{Mechanism}

Blunt thoracic aorta injury (BTAI) is a modern phenomenon due primarily to motor vehicle and motorcycle crashes. Burkhart reported that $72 \%$ of BTAI are caused by motor vehicle crashes (MVC), $20 \%$ caused by a pedestrian being struck by a car, and $8 \%$ caused by motorcycle crashes; de Metral et al. found that $90 \%$ were caused by MVC [1]. The traditional teaching is that head-on impact is predominately associated with BTAI, causing deceleration of the aorta at fixed location leading to an intimal or full-thickness tear. While this does account for almost half of the causative mechanism $(45 \%)$ in some series [2], a study from Britain indicated that side impacts may account for $44 \%$ of impacts compared with $29 \%$ of front impacts [2].

Blunt injury to the chest is typically conceptualized as being one of three types of injury: direct compression, shear, or crush type. Blunt thoracic aortic injury in particular is thought to occur secondary to the shear force that occurs between the rapidly decelerating aorta that is mobile and points of fixation. In support of this hypothesis, up to a $92 \%$ of BTAI occur at the aortic isthmus [3]. Autopsy studies have found a somewhat lower incidence of injury at the isthmus (58\%) while injuries in multiple locations (16\%), in the ascending aorta $(8 \%)$ and the distal descending aorta $(8 \%)$, were additional locations [4].

Blunt injury to the diaphragm is thought to be caused by rapid changes in intracavitary pressure. However, a recent database review also identified lateral mechanism and high 
changes in velocity as being significantly associated with diaphragm injury [5•]. Normal intraabdominal pressures range between 2 and $10 \mathrm{~cm} \mathrm{H}_{2} \mathrm{O}$ with a maximum of $100 \mathrm{~cm} \mathrm{H}_{2} \mathrm{O}$ with Valsalva. However, during blunt trauma, pressures can reach $1000 \mathrm{~cm} \mathrm{H}_{2} \mathrm{O}$ [6]. Similarly, blunt esophageal injuries, while rare, are hypothesized to occur secondary to rapid intraluminal pressure changes.

\section{Blunt Traumatic Aortic Injury (BTAI)}

\section{Epidemiology of Aortic Injury}

Injury to the aorta is the second most common cause of death after traumatic brain injury [7]. It is estimated that aortic injury causes 10-25\% of deaths due to MVC [8]. In one autopsy study, approximately $20 \%$ of fatalities from MVC had aortic injuries [9]. The incidence of death from aortic injury has not changed significantly in the recent past. Additionally, the age distribution of aortic injury significantly increases in younger patients, becoming more common the closer to the driving age of 16 . Incidence is significantly lower in the pediatric population [3].

Safety devices have been found to decrease the incidence of blunt thoracic aortic injury but do not have a significant impact on the mortality rate when they are present. In the previously mentioned autopsy study, the incidence of BTAI did not change despite a $10 \%$ increase in seat belt use during the study period. However, in a study examining the Pennsylvania Trauma Outcome Study database, the incidence of BTAI in MVC decreased from $1.3 \%$ without protective devices to $0.7 \%$ with seat belts. Additionally, the use of air bags without seat belts did not significantly decrease the incidence of BTAI versus no restraints (1.3 to $1.2 \%$ ), and the addition of air bags plus seat belts versus seat belts only did not change the BTAI incidence $(0.7$ and $0.7 \%)$ [10]. That said, this report concluded that while the use of restraint devices impacted the incidence of BTAI, it did not impact the rate of death per incident, i.e., the lethality of BTAI was similar.

Death from aortic injury occurs in three phases. Of those who die of aortic injury, 70-90\% die immediately at the scene and are not transported [11, 12]. Rapid exsanguination is the cause of death in these patients. Another $29 \%$ die within the first hour of reaching the hospital [2], typically of hemorrhage or multi-organ system trauma. Of those who survive to make it to the ICU, the in-hospital mortality is $44 \%$ [8]. Patients with BTAI typically have major associated injuries. Average ISS scores range from 34 to $40[13 \bullet \cdot, 14]$. Associated injuries included orthopedic $(81 \%)$, thoracic $(76 \%)$, abdomen (29\%), pelvic (15\%), and TBI (39\%) [14, 15]. Cause of death beyond the first $24 \mathrm{~h}$ is frequently due to the complications of multi-system trauma instead of an aortic injury.

\section{History and Physical Findings}

Motor vehicle crash is the most common cause of BTAI; however, assaults, auto versus pedestrian, falls, blast, and crush injury have also caused aortic injuries. As discussed in the above section, specifics of the mechanism of injury are important. Frontal impact collisions are a frequent but not exclusive cause of BTAI [16]; a more important factor is the amount of force transferred. This can be extrapolated from the degree of vehicle damage, the height of fall, etc. Additional information that should be obtained includes a history of intermittent paralysis or hemodynamic instability after the incident and the status of other patients present in the incident.

As with all patients with a significant mechanism of blunt trauma, a thorough secondary survey should be completed after the A, B, C's of ATLS have been performed. Degree of hemodynamic stability should be assessed and reassessed at regular intervals. Impressive findings such as visible flail chest, expanding hematoma at the thoracic outlet, palpable fractures of the sternum or thoracic spine, and external evidence of major trauma such as a steering wheel imprint on the chest should immediately alert the clinician to the possibility of BTAI. More subtle findings on physical exam include unequal blood pressures between the upper extremities or between the upper and lower extremities and an intrascapular murmur [17].

\section{Radiology}

As the initial radiological study in the majority of trauma patients, providers should be familiar with the welldescribed signs of aortic injury on a supine AP chest X-ray. These signs include widened mediastinum $(>8 \mathrm{~cm})$, left pleural effusion, downward displacement of the left bronchus $\left(>140^{\circ}\right.$ from trachea), deviation of the trachea to the right, shadowing or obliteration of the aortic knob contour, loss of the aortopulmonary window on lateral X-ray, loss of perivertebral pleural stripe, fracture of the first or second rib, and deviation of the nasogastric tube in the esophagus. In addition, patients with a higher suspicion for occult thoracic injury, as defined by the recently validated NEXUS chest criteria $[18 \cdot \bullet$ ] should receive computed tomography scanning with intravenous contrast. In patients with a moderate mechanism of injury, recent studies have indicated that whole body (or "pan") scanning is cost-effective and reduces mortality $[19,20]$. While the diagnosis of BTAI can be suggested by radiography, the trend for definitive diagnosis is toward CT angiography and away from aortography [21] (Table 1).

\section{Classification}

Classification of BTAI is based on characteristics seen on CT angiography. The classification system used in the guidelines 
Table 1 NEXUS criteria for CT scanning of the chest in blunt trauma

Age $>60$ years old

Rapid deceleration mechanism (e.g., fall $>20 \mathrm{ft}, \mathrm{MVC}>40 \mathrm{mph}$ )

Chest pain

Intoxication

Abnormal alertness/mental status

Distracting painful injury

Tenderness to palpation of chest wall

of the Society for Vascular Surgery (SVS) is most widely used (Table 2). These classifications become important as they predict rupture risk and help dictate management decisions. For instance, a recent study reviewing the natural history of class I and II injuries demonstrated that of 46 grade I injuries, two progressed on repeat imaging, half resolved, and the remaining $40 \%$ were stable [22].

\section{Initial Management}

As discussed above, those with aortic injuries who are fortunate enough to survive transport to the emergency department typically are severely injured. Initial management aimed at stabilizing the ABCs and identifying all other injuries should be performed.

The decision regarding the need for aortic repair, the timing of such repair, and even the technique of repair has been rapidly evolving and remains controversial. In part, this is due to not only the new techniques of endovascular repair but also a relative lack of understanding of the natural history of some injuries and the complexities of management of associated injuries. General guidelines have been developed by the major

Table 2 Classifications of blunt thoracic aortic injury

SVS classification

I. Intimal tear

II. Extramural hematoma

III. Pseudoaneurysm

IV. Transection

Presley Trauma Center Classification

Ia. Normal thoracic aorta on CT

Ib. Normal thoracic aorta on CT with mediastinal hematoma present

IIa. Intimal flap or pseudoaneurysm $<1 \mathrm{~cm}$

IIb. Intimal flap or pseudoaneurysm $<1 \mathrm{~cm}$ with mediastinal hematoma present

IIIa. $>1 \mathrm{~cm}$, easily identified, regular, well-defined pseudoaneurysm with intimal flap or thrombus

IIIb. $>1 \mathrm{~cm}$, easily identified, regular well-defined pseudoaneurysm with intimal flap or thrombus with mediastinal hematoma present

IV. Total aortic distruption; easily identified, irregular, poorly defined pseudoaneurysm with intimal flap or thrombus with mediastinal hematoma present trauma and vascular surgical organizations (EAST, SVS) [23, 25]. What follows is a summary of the key principles.

Patients with type I injury should be managed medically with impulse control, defined as $\mathrm{dP} / \mathrm{dt}$ [24••]. The SVS guidelines do not recommend specific therapeutic parameters, but goals of impulse control typically include a heart rate less than 100 and a systolic blood pressure less than $100 \mathrm{mmHg}$. Typically, the beta blocker esmolol at a starting dose of $50 \mathrm{mcg} /$ $\mathrm{kg} / \mathrm{min}$ is the best choice as it is rapidly titratable and controls both heart rate and blood pressure. Additional agents such as calcium channel blockers (diltiazem $5 \mathrm{mg} / \mathrm{h}$, titrated to maximum rate of $15 \mathrm{mg} / \mathrm{h}$ ) and vasodilators (nitroglycerin at $5 \mathrm{mcg} / \mathrm{minute}$, titrated by $5-10 \mathrm{mcg}$ every $10 \mathrm{~min}$ to a maximum of $400 \mathrm{mcg} / \mathrm{min}$ ) may be used to attain hemodynamic goals. These agents are preferred as they have a rapid onset and undergo rapid clearance; important properties as hemodynamic fluctuations are common in this frequently multiple injured population.

Patients with more severe injury (classes II-IV) should be evaluated for repair [11]. Issues that arise regarding repair include the suitability for repair, type of repair (open vs. endovascular), the timing of repair (early vs. delayed), the anatomy of the injury, and the management of associated injuries (especially TBI). In patients with concomitant BTAI and solid organ injury, a retrospective study from a single center were compared with patients with only BTAI and only solid organ injury [25]. This study found no difference in mortality, hospital length of stay, or blood transfusion between the groups and concluded that non-operative management of patients with grade I and II solid organ injuries can safely undergo operative correction of their aortic injuries. No data exists examining the safety of fixing orthopedic injuries in patients with BTAI.

Patients with relative contraindications to repair include those with devastating TBI, patients with significant infectious issues that preclude insertion of graft material, and patients with multiple injuries or co-morbidities who would be unlikely to survive any type of general anesthesia or operative procedure.

Patients who are in hemorrhagic shock from obvious aortic tear and exsanguinating thoracic bleed should be taken to the OR for emergent vascular control and aortic repair via open thoracotomy. While this continues to be the most rapid method of vascular control and the techniques of open aortic repair or graph replacement are well described, these patients often do poorly with mortality approaching $100 \%[1,2]$.

The ideal timing for repair of hemodynamically stable patients with higher grade BTAI remains controversial. Traditional dogma mandated early repair in what was felt to be a high-risk population. However, in 1998, Fabian et al. first published the success of impulse control in reducing rupture in patients with BTAI [26]. Since then, multiple authors have published their experience with both non-operative 
management and delayed intervention, particularly with grade I and II injuries [15, 23, 27, 28•].

Patients with grade III and IV injuries who are at high risk of rupture were recently reviewed [28•]. The authors performed a retrospective analysis of 49 patients with class III and IV BTAI and found that risk factors associated with rupture included a serum lactate level $>4 \mathrm{mM}$, mediastinal hematoma $>10 \mathrm{~mm}$, and a ratio of lesion to normal aorta diameter greater than 1.4 to 1 . The sensitivity of predicting aortic rupture in patients with two or more of these factors was $100 \%$ (specificity was $84 \%$ ), while no patients with fewer than two risk factors suffered a rupture. These factors should be taken into consideration when planning timing of repair [29॰].

With the presence of a TBI in conjunction with a BTAI, recent results from a similar retrospective review from the same group demonstrated a worsening of radiographic TBI in the cohort of patients that received fixation in the first $24 \mathrm{~h}$ after admission. This result was independent of fixation type or anti-coagulation used [30 ${ }^{\bullet}$. A possible etiology of this worsening mental status can be explained by clinically silent ischemia that manifests more prominently in patients with tenuous areas of brain tissue. A German group looked at patients undergoing elective TEVAR for non-traumatic disease and found that $63 \%(12 / 19)$ had new ischemic lesions numbering from one to six per patient [31]. It is hypothesized that emboli are disrupted by wire, catheter, or device manipulation in the diseased aorta. Whether these findings apply to patients with an otherwise disease free aorta has not been investigated; however, it does raise questions about how TEVAR may impact the neurologic status of patients with even mild neurologic derangements after trauma.

Patients with stable, high-grade aortic lesions should be treated with an endovascular covered stent graft according to SVS guidelines [11]. While the debate regarding open versus thoracic endovascular aortic repair (TEVAR) still has unanswered questions, enough data has accumulated in favor of TEVAR for the SVS to offer grade $2 \mathrm{C}$ guidelines recommending TEVAR over open thoracotomy. The evidence for this recommendation continues to accrue [32,33]. As an example of this data, these two meta-analyses found improved mortality and decreased complications with endovascular repair versus open. A randomized controlled trial has yet to be published.

One of the early concerns regarding the implantation of covered stent grafts into young trauma patients was the angulation of the aorta and the durability of these devices in this setting [34]. Second generation devices that are now FDA approved for BTAI have been validated in prospective trials. Two prospective non-randomized, multi-center trials confirmed the safety of Gore \& Associates C-TAG ${ }^{\circledR}$ device and Medtronic Inc.'s Valiant Captive ${ }^{\circledR}$ system. Both studies had similar results with universally successful placement, 30 -day morality around $8 \%$, and low device-related complications $[35,36]$.
Furthermore, these results compare favorably to the outcomes seen in with open repair. In a retrospective review of prospectively collected data, 56 patients undergoing open repair were compared to 50 patients who received TEVAR. Of note, the TEVAR group was statistically older, but all other characteristics including ISS were equivalent. Seventy percent of the patients receiving an open repair had complications compared to $48 \%$ of the TEVAR group. Additionally, when compared to the open cohort, the adjusted odds ratio of in-hospital morality was 0.33 in the TEVAR group, indicating a three times higher likelihood of dying with an open repair. The authors of this study concluded that TEVAR should be performed preferentially to open repair in appropriate patients $[37 \cdot \bullet]$.

\section{Management of the Left Subclavian Artery}

In the 2011 guidelines from the SVS, selective revascularization of the left subclavian artery (LSCA) was recommended. Statistically, LSCA coverage is required to allow for appropriate proximal landing site in approximately one of every three patients. For those who do require LSCA coverage, the decision to revascularize the LSCA is based primarily on the status of the ipsilateral vertebral artery. It is desirable to preserve ante grade flow in this artery to minimize the risk for stroke. Intraoperative angiography is the preferred method of expeditiously evaluating this attribute of the posterior circulation. Relative indications for revascularization include an atretic or hypoplastic artery or a circle of Willis which is discontinuous. Additionally, patients with spinal cord injuries in which preservation of collateral flow to the spine in vital should be considered for revascularization. Ultimately, the decision to revascularize should be individualized based on patient characteristics and provider comfort.

\section{Additional Management Decisions}

Despite the concerns of bleeding in a patient with multiple injuries including TBI, the decision to anticoagulate patients undergoing TEVAR is very much debated. In fact, the SVS guidelines suggest that a majority of committee members do anti-coagulate patients but at lower dose than typically administered. Decisions for anti-coagulation should be individualized. Routine placement of spinal drain placement is not advised. Because the average rate of spinal ischemia after TEVAR is approximately $3 \%$, spinal drainage is only indicated for patients who develop signs or symptoms of ischemia after stent graft placement. 


\section{Esophageal Injury}

Blunt esophageal rupture is extremely rare. There are no published guidelines to direct management though decisions typically follow similar algorithms for esophageal perforation due to any cause. Case reports combined with literature reviews still dominate the recent literature on this uncommon entity [38-40].

\section{Epidemiology}

Harborview Medical Center in Seattle reported two blunt esophageal injuries in 7 years out of a total of approximately 28,000 patients [41]. In a recent database review from Scotland, the incidence of blunt esophageal injury was 17 in 52, 887 trauma patients or $0.03 \%$. These patients were predominantly young men, and the mechanism was overwhelmingly motor vehicle crash or assault. Eighty-three percent were severely injured based on an ISS score of $>15$. In fact, the median ISS for this cohort was 41 . The in-hospital mortality was $82 \%$, due primarily to associated injuries [42•].

\section{Diagnosis}

Symptoms of esophageal injury include neck pain and pain with swallowing and drooling; however, patients can be asymptomatic particularly if their sensorium is altered. Signs of esophageal injury are not specific to that organ; they can be seen with many other types of injuries to the thorax. These signs include subcutaneous emphysema and cervical tenderness [43].

Because of the paucity of signs or symptoms unique to esophageal injury, diagnosis must be made with imaging. While the best test for diagnosing esophageal perforation is a probably fluoroscopic barium swallow, this test is often impossible to perform early in poly-trauma patients. Patients with pneumomediastinum noted on plain chest radiograph following blunt chest trauma should undergo a chest CT. The Harborview series mentioned above noted 136 cases of CT-diagnosed pneumomediastinum from blunt injury or which two had an esophageal injury $(1.5 \%$ incidence with pneumomediastnum) confirmed by esophagography and surgery. Of note, one patient over this 7-year time period and nearly 40,000 admissions had a blunt esophageal injury and no pneumomediastinum [40]. These authors believe that CT of the chest is an adequate screen examination, and only those with pneumomediastinum and a high suspicion of esophageal injury warrant an esophogram.

In 2010, a retrospective study of 897 blunt trauma patients with chest CT performed on initial assessment revealed an incidence of occult pneumomediastinum (oPNM) of $5.6 \%$. Occult pneumomediastinum was defined as mediastinal air seen on chest CT but not on initial chest X-ray. The authors further demonstrated that oPNM was not associated with increased mortality and importantly that in their cohort of 53 patients, no tracheal, bronchial, or esophageal injuries were diagnosed. Furthermore, the authors examined the seven patients in their registry since 1994 who had a tracheal, bronchial, or esophageal injury. None of the seven had an esophageal injury and of the four who did have intrathoracic bronchial injury, all had pneumomediastinum seen on chest X-ray. Finally, 11 of those patients with oPNM underwent repeat scanning of the chest. All patients had complete resolution of oPNM with the longest time between initial and follow up scan being $6 \mathrm{~h}$ [44].

\section{Management}

Patients with blunt esophageal injury should be approached similarly to patients with spontaneous or iatrogenic injury to the esophagus. Those without signs of sepsis and with a barium swallow showing a contained perforation can be managed with NPO status, intravenous antibiotics, and close monitoring. Nasogastric tubes should be avoided.

Patients who fail to improve with non-operative management or who have evidence of sepsis or have free extravasation of contrast on esophagram should be taken to the operating room for definitive repair [45]. Choice of incision (cervical, thoracotomy, or laparotomy) should be dictated by the location of the injury, concomitant injuries, and surgeon experience. Operative options include debridement and primary repair in patients who are hemodynamically stable with minimal contamination or resection and diversion with cervical esophagostomy in unstable patients and those with significant contamination. It should be noted that in our review of the literature, despite the proliferation of stent placement in patients with benign esophageal perforations, no reports of esophageal stent placement for blunt traumatic injury were identified.

\section{Diaphragmatic Injury}

Blunt injury to the diaphragm is, similar to injury of the aorta and esophagus, infrequent. One recent analysis of motor vehicle crash data found an incidence of $3.4 \%$ [1], consistent with the range typically reported in the literature of $1-8 \%$ [2]. However, similar to the other diseases, it is associated with an increased mortality, though analysis indicates that it may be a marker of injury severity rather than the direct cause of death. Lateral impact collisions are more likely to be associated with diaphragm rupture [5•]. Additionally, $90 \%$ of patients with diaphragm injury have associated injuries. Most of the recent literature on this topic relates to radiologic diagnosis rather than advances in management. 


\section{Diagnosis}

The diagnosis of blunt diaphragm injury can be challenging and can be missed on 12-63\% of CT scans [46]. Signs and symptoms are non-specific and frequently overshadowed by other injuries. Asymptomtic presentation is not uncommon.

Chest X-ray, which is ubiquitous in the trauma bay, is not sensitive enough to definitively rule out diaphragm injury. Classic signs such as nasogastric tube passing back above the diagram or the presence of an intrathoracic gastric bubble are unusual. CT is more sensitive than chest X-ray. Using newer, higher definition CT scanners and multi-planar reformations, a recent detection rate of $77 \%$ of surgically proven diaphragm injuries could be identified on pre-operative CT scan [47•]. Additionally, left-sided diaphragm injuries are more readily identified than right-sided injuries, likely due to the liver on the right side. There have been as many as 19 distinct radiological signs of diaphragm rupture described. In a recent review, those found to have high specificity and with the highest inter-rater reliability are the dependent viscera sign, organ herniation sign, discontinuity of diaphragm profile, and the collar sign. The dependent viscera sign is present when abdominal contents obliterate the posterior costophrenic sulcus allowing organs to lay against the posterior wall in the supine position. The organ herniation sign has a high sensitivity and specificity (60-90 and 94-100\%, respectively) [2]. Organs, most frequently the stomach on the left side, present through the rupture and can be seen in the chest. Similarly, the collar sign is seen when intraabdominal organs herniate into the chest and become trapped between surrounding diaphragm. This sign is most easily identified on coronal and sagittal reformations. Finally, the discontinuity of the diaphragm is the demonstration of termination of the musculotendious portion of the diaphragm, frequently with associated edema. Again, this sign is more sensitive when viewed on sagittal views. To reiterate, even with improvements in CT imaging, the sensitivity of CT scan is only able to identify three of every four injuries. Our approach is to perform diagnostic laparoscopy when we have a suspicion or concern for a diaphragmatic injury following blunt trauma, regardless of side, and when the patient is appropriately resuscitated and stable. The diaphragm injury itself is not an emergency unless bowel strangulation is a concern, but the injury should be recognized and repaired during the primary hospitalization. If an injury is found, laparoscopic or open repair is performed, based on surgeon preference and extent of the laceration.

\section{Management}

Given that such a high proportion of patients with diaphragm injury have significant associated injuries, unstable patients should be rapidly evaluated and treated for their life- threatening injuries. Should this require operative exploration of the chest or abdomen, intraoperative evaluation for diaphragm injury should be undertaken. If feasible, repair should be performed at the initial operation. For stable patients, appropriate CT scanning with multi-planar reprocessing should be performed. If signs concerning for a diaphragm rupture are identified, direct investigation of the diaphragm is the next step. This can be adequately achieved by a variety of approaches including laparoscopy, laparotomy, thoracoscopy, or thoracotomy. As surgeon comfort is increasing, laparoscopic repair has become more prevalent [48••]. Repair should be completed with a running, permanent suture.

\section{Conclusion}

The result of a recent review of the literature pertaining to blunt injury to the aorta, esophagus, and diaphragm point to a few major trends. Blunt thoracic aortic injury is undergoing a major paradigm shift regarding treatment. Recent guidelines have recommended endovascular repair over open repair. New generation devices which address concerns about the ability of stents to conform to the aortas of young trauma patients have been found to be safe and effective. Furthermore, as longer term outcome data becomes available, the endovascular approach has demonstrated few early complications, decreased mortality, and no significant long-term device-related complications. Meanwhile, the incidence of blunt esophageal continues to remain very low. While the mortality from these complex injuries also remains high, the low incidence precludes clinical trials to produce management advances. Specifically, the use of cover stents has not, to our knowledge, been applied to blunt esophageal injury. Finally, our knowledge regarding blunt diaphragm injury continues to evolve as diagnostic imaging modalities continue to improve. Right-sided injuries may be more common than previously believed, though left-sided injuries continue to predominate. The management of these injuries is increasingly being managed via minimally invasive techniques. As surgeons gain more experience with this approach, data regarding the optimal management may become available. Though blunt injuries to the aorta, esophagus, and diaphragm remain uncommon, providers should stay apprised of recent advances as the literature on these subjects continue to evolve.

\section{Compliance with Ethics Guidelines}

Conflict of Interest Drs. Lollar and Jurkovich declare that they have no conflicts of interest.

Human and Animal Rights and Informed Consent This article does not contain any studies with human or animal subjects performed by any of the authors. 


\section{References}

Papers of particular interest, published recently, have been highlighted as:

- Of importance

•- Of major importance

1. de Mestral C, Dueck A, Sharma SS, et al. Evolution of the incidence, management, and mortality of blunt thoracic aortic injury: a population based analysis. J Am Coll Surg. 2013;216:1110-5.

2. Richens D, Kotidis K, Neale M, et al. Rupture of the aorta following road traffic accidents in the United Kingdom 1992-1999. The results of the co-operative crash injury study. Eur J Cardiothorac Surg. 2003;23:143-8.

3. Watson J, Slaiby J, Toca MG, et al. 14 year experience with blunt thoracic aortic injury. J Vasc Surg. 2013;58:380-5.

4. Burkhart HM, Gomez GA, Jacobson LE, et al. Fatal blunt aortic injuries: a review of 242 autopsy cases. J Trauma. 2001;50:113-5.

5. Ryb GE, Dischinger PC, Ho S. Causation and outcomes of diaphragmatic injuries in vehicular crashes. J Trauma Acute Care Surg. 2013;74:835-8. Database review of 80 cases of diaphragm rupture demonstrating the association between lateral impact or change in velocity of $40 \mathrm{~km} / \mathrm{h}$ or greater and blunt injury to the diaphragm.

6. Bocchini G, Guida F, Sica G, et al. Diaphragmatic injuries after blunt trauma - still a challenge? Emerg Radiol. 2012;19:225-35.

7. Smith RS, Chang FC. Traumatic rupture of the aorta: still a lethal injury. Am J Surg. 1986;152:660-3.

8. Schulman CI, Carvajal D, Lopez PP, et al. Incidence and crash mechanisms of aortic injury during the past decade. J Trauma. 2007;62:664-7.

9. Kaytal D, McLellan BA, Brenneman FD, et al. Lateral impact motor vehicle collisions: significant cause of blunt traumatic rupture of the thoracic aorta. J Trauma. 1997;42:760-72.

10. Laurence G, Grim R, Bell T, et al. Impact of seat belt use and airbag deployment on blunt thoracic aortic injury — brief report. Am Surg. 2013;79:E335-336.

11. Parmley LF, Mattingly TW, Manion WC, et al. Nonpenetrating traumatic injury to the aorta. Circulation. 1958;17:1086-101.

12. Smith RS, Chang FC. Traumatic rupture of the aorta. Am J Surg. 1986;152:660-3.

13.• Azizzadeh A, Ray HM, Dubose JJ. Outcomes of endovascular repair for patients with blunt traumatic aortic injury. J Trauma Acute Care Surg. 2014;76:510-6. Single- center experience with 82 consecutive patients with BTAI who underwent endovascular repair with high success rate and low complications and secondary procedures.

14. Arthurs ZM, Starnes BW, Sohn VY, et al. Functional and survival outcomes in traumatic blunt thoracic aortic injuries: an analysis of the National Trauma Databank. J Vasc Surg. 2009;49:988-94.

15. Di Eusanio M, Folesani G, Berretta P, et al. Delayed management of blunt thoracic aortic injury: open versus endovascular repair. Ann Thorac Surg. 2013;95:1591-7.

16. Fabian TC, Richardson JD, Croce MA, et al. Prospective study of blunt aortic injury: multicenter trial of the American Association for the Surgery of Trauma. J Trauma. 1997;42:374-80.

17. Mattox KL, Moore EE, Feliciano D. Chapter 26, Heart and Thoracic Vascular Injuries. Trauma 7th ed. McGraw Hill Medical, 2013, ISBN 978-0-07-166351-7

18.• Rodriguez RM, Anglin D, Langdorf MI, et al. NEXUS Chestvalidation of a decision instrument for selective chest imaging in blunt trauma. JAMA Surg. 2013;148(10):940-6. Validation of the NEXUS Chest instrument in 9905 patients demonstrating a sensitivity of $99.7 \%$ and negative predictive value of $99.9 \%$ for predicting clinically major thoracic injury.

19. Lee WS, Parks NA, Garcia A, et al. Pan computed tomography versus selective computed tomography in stable, young adults after blunt trauma with moderate mechanism: a cost-utility analysis. J Trauma Acute Care Surg. 2014;77:527-33.

20. Caputo ND, Stahmer C, Lim G, et al. Whole body computed tomography scanning leads to better survival as opposed to selective scanning in trauma patients: systematic review and meta-analysis. J Trauma Acute Care Surg. 2014;77:534-9.

21. Demetriades D, Velmahos GC, Scalea TM, et al. Diagnosis and treatment of blunt thoracic aortic injury: changing perspectives. J Trauma. 2008;64:1415-9.

22. Osgood MJ, Heck JM, Rellinger EJ, et al. Natural history of grade III blunt traumatic aortic injury. J Vasc Surg. 2010;59:334 42.

23. Nagy K, Fabian T, Rodman G. Guidelines for the diagnosis and management of blunt aortic injury: an EAST practice management guidelines work group. J Trauma. 2000;48(6):1128-43.

24.• Lee WA, Matsumura JS, Mitchell RS, et al. Endovascular repair of traumatic thoracic aortic injury: clinical practice guidelines of the Society for Vascular Surgery. J Vasc Surg. 2011;53:187-92. Only set of recent guidelines for management of patients with BTAI from a major surgical society. Though evidence is weak, the group recommends endovascular repair over open thoracotomy.

25. Santaniello JM, Miller PR, Croce MA, et al. Blunt aortic injury with concomitant intra-abdominal solid organ injury: treatment priorities revisited. J Trauma. 2002;53:442-5.

26. Fabian TC, Davis KA, Gavant ML, et al. Prospective study of blunt aortic injury and helical CT is diagnostic and antihypertensive therapy reduces rupture. Am Surg. 1998;227:666-77.

27. Demetriades D, Velmahos GC, Scalea TM, et al. Blunt traumatic thoracic aortic injuries: early or delayed repair - results of an American Association for the Surgery of Trauma Prospective Study. J Trauma. 2009;66:967-73.

28. Paul JS, Neideen T, Tutton S, et al. Minimal aortic injury after blunt trauma: non-operative management is safe. J Trauma. 2011;71: 1519-23. Retrospective, single- center review of 47 patients with BTAI, $32 \%$ of whom were managed without repair and had fewer ventilator days, hospital days and no adverse outcomes.

29. Harris DG, Rabin J, Kufera JA, et al. A new aortic injury score predicts early rupture more accurately than clinical assessment. J Vasc Surg. 2014. in press: 1-7. Retrospective, single- institution study of 49 stable patients with Grade III or IV BTAI analyzing the risk factors in the cohort of 18 patients who subsequently ruptured.

30. Rabin J, Harris DG, Crews GA. Early aortic repair worsens concurrent traumatic brain injury. Ann Thorac Surg. 2014;98:46-52. Retrospective, single- institution, analysis demonstrating worse neurologic outcomes in patients with BTAI and TBI who underwent early repair of their aortic injury.

31. Kahlert P, Eggebrecht H, Janosi RA, et al. Silent cerebral ischemia after thoracic endovascular aortic repair: a neuroimaging study. Ann Thorac Surg. 2014;98:53-8.

32. Tang GL, Tehrani HY, Usman A, et al. Reduced mortality, paraplegia, and stroke with stent graft repair of blunt thoracic aortic transection: a modern meta-analysis. J Vasc Surg. 2008;47:671-5.

33. Xenos ES, Abedi NN, Davenport DL, et al. Meta-analysis of endovascular vs open repair for traumatic descending thoracic aortic rupture. J Vasc Surg. 2008;48:1343-51.

34. Adams JD, Kern JA. Blunt thoracic aortic injury: current issues and endovascular treatment paradigms. Endovascular Today 2014; 38 42.

35. Farber MA, Giglia JS, Starnes BW, et al. Evaluation of the redesigned conformable GORE TAG thoracic endoprosthesis for traumatic aortic transection. J Vasc Surg. 2013;58:651-8. 
36. Khoynezhad A, Azizzadeh A, Donayre CE, et al. Results of a multicentered, prospective trial of thoracic endovascular aortic repair for blunt thoracic aortic injury (RESCUE trial). J Vasc Surg. 2013;57:899-905.

37.• Azizzadeh A, Charlton-Ouw KM, Chen Z, et al. An outcome analysis of endovascular versus open repair of blunt traumatic aortic injuries". J Vasc Surg. 2013;57:108-15. Retrospective analysis of prospective database comparing 106 patients with BTAI, 50 with endovascular repair and 56 who received open repair. This series demonstrated improved mortality and decreased complications in the endovascular group versus the open group.

38. Delos Reyes AP, Clancy C, Lach J, et al. Conservative management of esophageal perforation after a fall. Int J Surg Case Rep. 2013;4: 550-3.

39. Uygun I, Otcu S, Aydogdu B, et. al, Total esophageal avulsion at the esophagogastric junction after blunt trauma. Case Reports in Surg. $2013 ; 1-4$

40. Sogut O, Cevik M, Boleken ME. Pneumomediastinum and subcutaneous emphysema due to blunt neck injury: a case report and review of the literature. J Pak Med Assoc. 2011;61(7):702-4.

41. Dissanaike S, Shalhub S, Jurkovich GJ. The evaluation of pneumomediastinum in blunt trauma patients. J Trauma. 2008;65: 1340-5.

42. Skipworth RJE, McBride O, Kerssens JJ, et al. Esophagogastric trauma in Scotland. World J Surg. 2012;36:1779-84. Population based study of over 50,000 trauma patients over 10 years in Scotland the incidence of blunt and penetrating esophageal and gastric injuries and identification of some predisposing risk factors.

43. Mattox KL, Moore EE, Feliciano D. Chapter 25, Lung, Trachea and Esophagus. Trauma 7th ed. McGraw Hill Medical, 2013, ISBN 978-0-07-166351-7

44. Rezende-Neto JB, Hoffman J, Mahroos MA, et al. Occult pneumomediastinum in blunt chest trauma: clinical significance. Injury. 2010;41:40-3.

45. Ivatury RR, Moore FA, Biffl W, et al. Oesophageal Injuries: Position Paper, WSES, 2013. World J Emergency Surg. 2014;9(9):1-7.

46. Panda A, Kumar A, Gamanagatti S, et al. Traumatic diaphragmatic injury: a review of CT signs and the difference between blunt and penetrating injury. Diagn Interv Radiol. 2014;20:121-8.

47. Hammer MM, Flagg E, Mellnick VM, et al. Computed tomography of blunt and penetrating diaphragmatic injury: sensitivity and interobserver agreement of CT signs. Emerg Radiol. 2014;21:143-9. A novel retrospective review by three indedendent clnicians of abdominal CT done for blunt or penetrating trauma in 84 patients who were eventually proven at operation to have a diaphragmatic injury. Twelve signs of diaphragm injury were examined.

48.• Ties JS, Peschman JR, Moreno A, et al. Evolution in the management of traumatic diaphragmatic injuries: a multicenter review. J Trauma Acute Care Surg. 2014;76:1024-8. Retrospective review of patients with diaphragmatic injuries over 15 years demonstrating a trend toward diagnosis with $\mathrm{CT}$ and repair with laparoscopy. 